subregions with differences in HER2 status be scored separately. ${ }^{6}$ Further work is needed to define the most revealing testing parameters with respect to prognosis, trastuzumab response, and chemotherapy response.

\section{Disclosure/conflict of interest}

The authors declare no conflict of interest.

Martin C Chang $\mathbf{1 , 2}$
${ }^{1}$ Department of Pathology and Laboratory Medicine,
Mount Sinai Hospital, Toronto, ON, Canada;
${ }^{2}$ Department of Laboratory Medicine and
Pathobiology, University of Toronto, Toronto,
ON, Canada
E-mail: mchang2@mtsinai.on.ca

\section{References}

1 Seol H, Lee HJ, Choi Y, et al. Intratumoural heterogeneity of HER2 gene amplification in breast cancer: its clinicopathological significance. Mod Pathol 2012;25: 938-948.

2 Bartlett AI, Starcynznski J, Robson T, et al. Heterogeneous HER2 gene amplification: impact on patient outcome and a clinically relevant definition. Am J Clin Pathol 2011;136:266-274.

3 Dowsett M, Procter M, McCaskill-Stevens W, et al. Disease-free survival according to degree of HER2 amplification for patients treated with adjuvant chemotherapy with or without 1 year of trastuzumab: the HERA trial. J Clin Oncol 2009;27:2962-2969.

4 Paik S, Bryant J, Tan-Chiu E, et al. HER2 and choice of adjuvant chemotherapy for invasive breast cancer: National Surgical Adjuvant Breast and Bowel Project Protocol B-15. J Natl Cancer Inst 2000;92:1991-1998.

5 Chang MC, Malowany JI, Mazurkiewicz J, et al. 'Genetic heterogeneity' in HER2 testing by fluoresence in situ hybridization: a study of 2522 cases. Mod Pathol 2012;25:683-688.

6 Vance GH, Barry TS, Blook KJ, et al. Genetic heterogeneity in HER2 testing in breast cancer: panel summary and guidelines. Arch Pathol Lab Med 2009;133:611-612.

\title{
Reply to 'Intratumoral heterogeneity of HER2 gene amplification in breast cancer: its clinicopathological significance'
}

\author{
Modern Pathology (2013) 26, 610-611; doi:10.1038/modpathol.2013.38
}

To the editor: We read with great interest the comments by Arena et al. and Chang in reference to our paper, 'Intratumoral heterogeneity of HER2 gene amplification in breast cancer: its clinicopathological significance'. ${ }^{1}$ Their letters focused on different issues of the HER2 intratumoral heterogeneity in breast cancer.

Arena et al. questioned about the best way to write HER2 reports for the clinician and suggested that HER2 analytical report should be completed with a critical evaluation of the results about HER2 genetic heterogeneity. Although the clinical relevance of HER2 genetic heterogeneity is not established in breast cancer, we agree that HER2 in situ hybridization report should include not only overall average ratio of HER2/CEP17 and average HER2 gene copy number, but also information about HER2 genetic heterogeneity. However, there are some issues to be addressed in the definition of HER2 genetic heterogeneity proposed by 2009 College of American Pathologists expert panel, which indicates the presence of tumor cells with HER2/CEP17 signal ratios $>2.2$ (or $>6$ HER2 signals per cell when using a probe for HER2 only) in $5-50 \%$ of the tumor cells tested. ${ }^{2}$ If 20 cells are counted and 1 tumor cell is identified with a HER2/CEP17 > 2.2, the tumor is diagnosed to have HER2 genetic heterogeneity. However, a recent study revealed that the tumor cells with 3:1 HER2/CEP17 ratio, which may reflect technical issues, were determining factor for heterogeneity in $46 \%$ of heterogeneous cases. ${ }^{3}$ Furthermore, Allison et al. ${ }^{4}$ reported that the ratio criteria and the criteria based on HER2 signals per cell for definition of HER2 genetic heterogeneity were not equivalent and the ratio-based definition resulted in large numbers of non-amplified cases being classified as heterogeneous. Thus, to avoid artifactual heterogeneity caused by technical issues, such as nuclear truncation and inadequate hybridization, cutoff values of percentage and cell ratio for HER2 genetic heterogeneity need to be validated. Furthermore, the number of cells to be counted and the fields to be selected for counting should be clearly defined through robust evidence.

HER2 intratumoral heterogeneity appears as two forms; distinct clusters of amplified cells and admixture of amplified and non-amplified cells. Distinct HER2 amplified clones in a non-amplified tumor, which was defined as HER2 regional heterogeneity in our study, should be scored separately, as proposed previously. ${ }^{2,5}$ HER2 regional heterogeneity can be assessed by scanning the entire tumor section before selection of fields to be counted and matching with HER2 immunohistochemistry (IHC). If the tumor has differentially amplified or stained area, the regions should be included in the counting. From this point of view, silver in situ hybridization has an advantage to evaluate HER2 regional 
heterogeneity, because it can be easily matched with HER2 IHC slide under light microscope. We agree to Arena et al. that detailed report of HER2 IHC is the simplest way to report HER2 regional heterogeneity. As shown in our study, heterogeneous HER2 expression was well matched with HER2 regional or genetic heterogeneity.

The main issue pointed out by Chang is the prognostic significance of HER2 intratumoral heterogeneity. He argued that the conflicting prognostic effect of HER2 intratumoral heterogeneity is related to patient treatment. We agree that different treatment may account for different outcome, as shown in the study by Bartlett et al. ${ }^{5}$ For survival analysis, they used TEAM (Tamoxifen vs Exemestane adjuvant Multicentre) pathology study composed of estrogen receptor-positive early breast cancers, treated with adjuvant endocrine therapy (exemestane versus tamoxifen), but not with chemotherapy. ${ }^{6}$ However, tumors with heterogeneous HER2 amplification cannot be equated with those with borderline/low HER2 amplification. Although HER2 genetic and regional heterogeneity was more common in tumors with equivocal/low-grade amplification, they were found in 23 and $38 \%$ of tumors with equivocal/lowgrade amplification in our study. Thus, comparison of our study with the HERA trial ${ }^{7}$ is not reasonable. We admit that our study has a limitation for survival analysis due to small number of heterogeneous cases. Further large-scaled, well-designed studies will be needed to find clinically relevant definition of HER2 intratumoral heterogeneity and to establish its prognostic significance and predictive value for HER2 targeted therapy.

\section{Disclosure/conflict of interest}

The authors declare no conflict of interest.
Hee Jin Lee ${ }^{1}$ and So Yeon Park ${ }^{1,2}$

${ }^{1}$ Department of Pathology, Seoul National University Bundang Hospital, Seongnam, Gyeonggi, Korea; ${ }^{2}$ Department of Pathology, Seoul National University College of Medicine, Seoul, Korea E-mail: sypmd@snu.ac.kr

\section{References}

1 Seol H, Lee HJ, Choi Y, et al. Intratumoral heterogeneity of HER2 gene amplification in breast cancer: its clinicopathological significance. Mod Pathol 2012; 25:938-948.

2 Vance GH, Barry TS, Bloom KJ, et al. Genetic heterogeneity in HER2 testing in breast cancer: panel summary and guidelines. Arch Pathol Lab Med 2009;133: 611-612.

3 Layfield LJ, Schmidt RL. HER2/neu gene amplification heterogeneity: the significance of cells with a 3: 1 HER2/ CEP17 ratio. Appl Immunohistochem Mol Morphol 2012;20:543-549.

4 Allison KH, Dintzis SM, Schmidt RA. Frequency of HER2 heterogeneity by fluorescence in situ hybridization according to CAP expert panel recommendations: time for a new look at how to report heterogeneity. Am J Clin Pathol 2011;136:864-871.

5 Bartlett AI, Starcyznski J, Robson T, et al. Heterogeneous HER2 gene amplification: impact on patient outcome and a clinically relevant definition. Am J Clin Pathol 2011;136:266-274.

6 Bartlett JM, Brookes CL, Robson T, et al. Estrogen receptor and progesterone receptor as predictive biomarkers of response to endocrine therapy: a prospectively powered pathology study in the Tamoxifen and Exemestane Adjuvant Multinational trial. J Clin Oncol 2011;29:1531-1538.

7 Dowsett M, Procter M, McCaskill-Stevens W, et al. Disease-free survival according to degree of HER2 amplification for patients treated with adjuvant chemotherapy with or without 1 year of trastuzumab: the HERA Trial. J Clin Oncol 2009;27:2962-2969.

\title{
DDIT3, STT3A (ITM1), ARG2 and FAM129A (Niban, C1orf24) in diagnosing thyroid carcinoma: variables that may affect the performance of this antibody-based test and promise
}

\author{
Modern Pathology (2013) 26, 611-613; doi:10.1038/modpathol.2012.212
}

To the editor: We have read with special interest, the paper by Sigstad et $a l^{1}$ published in a recent issue of Modern Pathology (25: 537-547, 2012). The authors sought to confirm whether ITM1 (alias STT3A), C1orf24 (aliases FAM129A and Niban), DDIT3 (alias GADD153) and ARG2 could discriminate follicular thyroid carcinoma (FTC) from follicular thyroid adenoma (FTA). In their study, they were not able to use these markers to discriminate between FTC and FTA.
In our previous work we found that these markers would discriminate between FTA and FTC with high sensitivity and specificity. Custom antibodies for ITM1 and C1orf24 and commercially available ARG2 and DDIT3 antibodies were tested on thyroid lesions commonly diagnosed as indeterminate by fine-needle aspiration biopsy. We showed that immunohistochemistry (IHC) was more sensitive than quantitative PCR in detecting thyroid carcinomas, and that the combination of four markers was 